\title{
(6) OPEN ACCESS \\ Genetic testing and blood biomarkers in paediatric pulmonary hypertension. Expert consensus statement on the diagnosis and treatment of paediatric pulmonary hypertension. The European Paediatric Pulmonary Vascular Disease Network, endorsed by ISHLT and DGPK
}

\author{
Joseph Pattathu, ${ }^{1}$ Matthias Gorenflo, ${ }^{1}$ Anne Hilgendorff, ${ }^{2}$ Juha W Koskenvuo, ${ }^{3,4}$ \\ Christian Apitz, ${ }^{5}$ Georg Hansmann, ${ }^{6}$ Tero-Pekka Alastalo ${ }^{4,7}$
}

For numbered affiliations see end of article.

\section{Correspondence to} Dr. Tero-Pekka Alastalo, Department of Paediatric Cardiology; University Hospital Helsinki; University of Helsinki; Paediatric Research Laboratory, Biomedicum Helsinki, Tukholmankatu 8, Helsinki 00290, Finland; tero-pekka. alastalo@helsinki.fi

This article is a product of the writing group of the European Paediatric Pulmonary Vascular Disease (PVD) Network (Writing Group Chair: G. Hansmann, Co-Chair: C. Apitz). DGPK, German Society of Paediatric Cardiology; ISHLT, International Society of Heart and Lung Transplantation.

Received 26 November 2014 Revised 26 January 2015 Accepted 13 February 2015

\section{CrossMark}

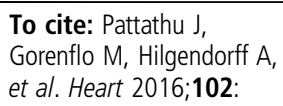

\section{ABSTRACT}

Childhood-onset pulmonary arterial hypertension (PAH) is considered complex and multifactorial, with relatively poor estimates of the natural history of the disease. Strategies allowing earlier detection, establishment of disease aetiology together with more accurate and sensitive biomarkers could enable better estimates of prognosis and individualise therapeutic strategies. Evidence is accumulating that genetic defects play an important role in the pathogenesis of idiopathic and hereditary forms of PAH. Altogether nine genes have been reported so far to be associated with childhood onset PAH suggesting that comprehensive multigene diagnostics can be useful in the assessment. Identification of disease-causing mutations allows estimates of prognosis and forms the most effective way for risk stratification in the family. In addition to genetic determinants the analysis of blood biomarkers are increasingly used in clinical practice to evaluate disease severity and treatment responses. As in genetic diagnostics, a multiplex approach can be helpful, as a single biomarker for PAH is unlikely to meet all requirements. This consensus statement reviews the current evidence for the use of genetic diagnostics and use of blood biomarkers in the assessment of paediatric patients with PAH.

\section{INTRODUCTION}

During the last decade, advances in genomic technologies have accelerated genetic research and broadened our understanding of basic human biology. Using genomics approaches to study wellcharacterised patient cohorts has revealed the molecular genetic background of many human diseases. Fast accumulation of knowledge on clinically relevant genomic variants has started to reshape clinical practices in several fields of medicine. These include hereditary cardiovascular diseases such as cardiomyopathies and channelopathies, where thousands of mutations in over 100 genes have been found. In these disorders the use of genetic testing is becoming a standard procedure in the diagnostic workup. Several international guidelines supporting the use of genetic testing have been published. ${ }^{1}$ It has been shown that these approaches can save costs by enabling rational follow-up focused on family members with an identified risk. ${ }^{2}$ Although genetic testing has not been widely applied to patients with familial pulmonary arterial hypertension (HPAH, hereditary forms of $\mathrm{PAH}$ ) or idiopathic $\mathrm{PAH}$ (IPAH), there is increasing evidence for its usefulness in the diagnostic workup. Other strategies to individualise patients' treatments and follow-up care involve the use of blood biomarkers. Biomarkers are suggested as potential clinical end points that can optimise the assessment and treatment of patients. This consensus statement reviews the current evidence for the use of genetic diagnostics and blood biomarkers in the assessment of paediatric patients with pulmonary arterial hypertension (PAH) with respect to diagnosis, prognosis and treatment.

\section{METHODS}

The recommendations given in table 1 relate to the grading system currently suggested by the European Society of Cardiology and the American Heart Association, and was based on paediatric data only (class, level of evidence). The grading and voting process within the writing group is outlined in the executive summary ${ }^{31}$ of this online supplement. Computerised searches of the PubMed/MEDLINE bibliographic database from 1990 to January 2015 were conducted. The developer searched using the terms 'genetics of pulmonary hypertension', 'paediatric pulmonary hypertension', 'genetic diagnostics in pulmonary hypertension', 'genetics of childhood-onset pulmonary hypertension', 'biomarkers of pulmonary hypertension', 'BNP and children', 'uric acid and children', 'circulating endothelial cells and children', 'biomarkers in children with pulmonary hypertension', 'circulating endothelial cells and pulmonary hypertension', 'BNP and pulmonary hypertension', 'uric acid and pulmonary hypertension'.

\section{Genetics of PAH}

To date, there are at least eight genes associated with HPAH and IPAH (table 2). From these genes, bone morphogenetic protein receptor 2 (BMPR2), 
Table 1 Recommendations on the use of genetic testing and biomarkers in children with pulmonary hypertension

\begin{tabular}{|c|c|c|}
\hline Genetics/genetic testing/blood biomarker & COR & LOE \\
\hline $\begin{array}{l}\text { Genetic counselling is recommended for families with children suffering from severe genetic disorder. This } \\
\text { includes children with IPAH or HPAH }\end{array}$ & I & B \\
\hline $\begin{array}{l}\text { Families of patients with syndromes associated with PAH should be educated on the symptoms of PAH and } \\
\text { recommended to seek clinical evaluation if the child should develop symptoms of PAH }\end{array}$ & I & C \\
\hline $\begin{array}{l}\text { It is recommended to screen asymptomatic PAH mutation carriers, also children, with serial echocardiograms, } \\
\text { and potentially other non-invasive studies }\end{array}$ & I & C \\
\hline $\begin{array}{l}\text { Genetic testing for PAH-associated genes such as ACVRL1, BMPR2, CAV1, ENG and KCNK3 can be useful for } \\
\text { children with PAH of unknown cause to allow definition of aetiology, estimation of prognosis and identification } \\
\text { of family members at risk, and to inform family planning }\end{array}$ & Ila & C \\
\hline $\begin{array}{l}\text { Genetic testing of first-degree relatives of an index patient with PAH and a known disease-causing mutation can } \\
\text { be useful for risk stratification and rationalising surveillance }\end{array}$ & Ila & C \\
\hline $\begin{array}{l}\text { Genetic testing for mutations in EIF2AK4 gene should be considered for children with suspicion of PVOD to allow } \\
\text { for definition of aetiology and identification of family members at risk, and to inform family planning }\end{array}$ & Ila & C \\
\hline $\begin{array}{l}\text { Family members of patients with HPAH who develop new cardiorespiratory symptoms should be evaluated } \\
\text { immediately for PAH }\end{array}$ & I & C \\
\hline $\begin{array}{l}\text { Comprehensive NGS panels targeting all known genetics in PAH can be considered to maximise diagnostic } \\
\text { efficacy and increase cost-effectiveness in genetic diagnostics }\end{array}$ & Ilb & C \\
\hline $\begin{array}{l}\text { Genetic testing for PAH-associated genes can be considered for patients with CHD and 'out of proportion' PAH } \\
\text { (eg, PAH with small atrial shunt) }\end{array}$ & Ilb & C \\
\hline $\begin{array}{l}\text { Measurement of natriuretic peptides BNP or NT-proBNP is recommended to evaluate disease severity, progression } \\
\text { and treatment response in patients with } \mathrm{PH}^{20-22}\end{array}$ & 1 & B \\
\hline It is uncertain whether analysis of uric acid levels can be used to evaluate disease severity & Ilb & C \\
\hline $\begin{array}{l}\text { Analysis of circulating endothelial cells can be useful to stratify operative risk, to evaluate for progression of } \mathrm{d} \\
\text { and/or response to therapy in children with PAH }\end{array}$ & Ila & B \\
\hline
\end{tabular}

ACVRL1 (ALK1) activin-like kinase-type 1; BMPR2, bone morphogenetic protein receptor 2; BNP, brain natriuretic peptide; CAV1, caveolin 1; CHD, congenital heart disease; COR, class of recommendation; EIF2AK4, eukaryotic translation initiation factor 2- $\alpha$ kinase 4; ENG, endoglin; HPAH, hereditary pulmonary hypertension; IPAH, idiopathic pulmonary arterial hypertension; KCNK3, potassium channel subfamily K, member 3; LOE, level of evidence; NGS, next generation sequencing; NT-proBNP, N-terminal fragment of probrain natriuretic peptide; PH, pulmonary hypertension; PVOD, pulmonary veno-occlusive disease; Definitions for COR and LOE classes can be found in our executive summary table 1 (LINK).

is the major gene associated with HPAH and IPAH. Today over 300 BMPR2 mutations have been identified in patients with PAH. BMPR2 mutations are estimated to account for approximately $75 \%$ of patients with HPAH and up to $25 \%$ of IPAH cases. ${ }^{3}$ The penetrance of the disease associated with BMPR2 mutations has originally been considered low. These observations have been questioned as disease manifestations can take 75 years and phenotypical variability exists. Supporting evidence for higher penetrance was established through the Vanderbilt Pulmonary Hypertension Registry where overall penetrance was $27 \%$ (female $42 \%$ and the male $14 \%$ ). ${ }^{4}$ Over $40 \%$ penetrance and high proportion of mutation positive individuals among patients with HPAH demonstrates that PAH is a genetic disease comparable with other forms of genetic diseases, i.e., cardiomyopathies and channelopathies. BMPR2 mutations are associated with childhood-onset PAH (table 2). ${ }^{5} 6$

PAH associated with hereditary haemorrhagic telangiectasia (HHT) has been identified in multiple studies to associate with pathogenic mutations in the ACVRL1 gene and in some occasions with Endoglin (ENG), a type III TGF- $\beta$ receptor. ${ }^{7}$ ACVRL1 mutations can also be identified in patients with PAH with no features of HHT. SMAD9 mutations have been suggested as a genetic cause of PAH although existing evidence is
Table 2 Genes associated with childhood-onset pulmonary hypertension

\begin{tabular}{llll}
\hline Gene & Form of PH & $\begin{array}{l}\text { Affecting } \\
\text { children }\end{array}$ & $\begin{array}{l}\text { High evidence of } \\
\text { pathogenicity }\end{array}$ \\
\hline ACVRL1 (ALK1) & IPAH, HPAH & $\checkmark$ & $\checkmark$ \\
BMPR1B & IPAH & $\checkmark$ & \\
BMPR2 & IPAH, HPAH & $\checkmark$ & $\checkmark$ \\
CAV1 & IPAH, HPAH & $\checkmark$ & $\checkmark$ \\
EIF2AK4 & PVOD & $\checkmark$ & $\checkmark$ \\
ENG & IPAH & $\checkmark$ & \\
KCNK3 & IPAH, HPAH & $\checkmark$ & $\checkmark$ \\
NOTCH3 & IPAH & $\checkmark$ & \\
SMAD9 & IPAH & $\checkmark$ &
\end{tabular}

High evidence of pathogenicity requires evidence for positive family segregation between genotype and phenotype or multiple reports supporting causative role in human disease together with additional evidence such as functional studies. ACVRL1 (ALK1) activin-like kinase-type 1; BMPR1B, bone morphogenetic protein receptor 1B; BMPR2, bone morphogenetic protein receptor 2; CAV1, caveolin 1; EIF2AK4, eukaryotic translation initiation factor 2- $\alpha$ kinase 4; ENG, Endoglin; HPAH, hereditary pulmonary hypertension; IPAH, idiopathic pulmonary arterial hypertension; KCNK3, potassium channel subfamily K, member 3; NOTCH3, neurogenic locus notch homologue protein 3 ; $\mathrm{PH}$, pulmonary hypertension; PVOD, pulmonary veno-occlusive disease; SMAD9, Smad family member 9. 
mainly based on experimental data derived from functional in vitro and animal studies. ${ }^{8}$ Two missense mutations in another bone morphogenetic protein (BMP) signaling associated gene, BMPR1B, were identified in children with IPAH. ${ }^{9}$ An exome sequencing analysis of a three-generation family with multiple family members presenting with PAH revealed CAV1 as a novel gene associated with $\mathrm{PAH}$. Another exome sequencing study on a family with multiple affected individuals revealed a missense mutation in a potassium channel gene KCNK3. ${ }^{10} \mathrm{~A}$ recent report suggested that mutations in the NOTCH3 gene could also cause childhood-onset IPAH. ${ }^{11}$ A recent breakthrough in pulmonary veno-occlusive disease (PVOD) has revealed that mutations in the EIF2AK4 gene play a major role in hereditary forms of PVOD. ${ }^{12}$ Genetic testing for mutations in EIF2AK4 gene should be considered for children with suspicion of PVOD. To date the evidence for causality in human IPAH and $\mathrm{HPAH}$ is considered significant for the following genes: ACVRL1, BMPR2, CAV1, ENG and KCNK3, whereas the role of BMPR1B, NOTCH3 and SMAD9 is still unconfirmed (table 2).

There is evidence for genotype-phenotype correlations in PAH. Multiple studies from different registries have implicated that carriers of a BMPR2 mutation, irrespective of family history, develop PAH at a younger age than mutation negative individuals. ${ }^{13-16}$ Furthermore, patients with the hereditary form of PAH developed higher mean pulmonary arterial pressure (PAP), lower cardiac index, higher pulmonary vascular resistance (PVR), and were more likely to be treated with parenteral prostanoid therapy or lung transplant when compared with patients with idiopathic disease. Both children and adults with PAH in association with a BMPR2 mutation are less likely to respond to vasodilators and are unlikely to benefit from treatment with calcium channel blockers. Symptomatic patients with HPAH carrying ACVRL1 mutations, most without HHT, have an earlier age of disease onset and show more rapid disease progression than patients with BMPR2 mutations. ${ }^{16}$ A recent study on 54 children with IPAH and HPAH revealed that 25 individuals (46\%) were positive for either BMPR2 (18 patients; 33\%) or ACVRL1 (7 patients; 13\%) mutations. ${ }^{6}$ In this study the 5 -year survival was significantly lower in patients tested positive for BMPR2 and ACVRL1 mutations. The study outcome correlated well with previous studies showing earlier onset and more severe phenotype in mutation positive patients. These studies suggest that patients with PAH carrying the BMPR2 or ACVRL1 mutation should be considered for more aggressive treatment strategies.

PAH is a rare complication in many genetic disorders such as Down syndrome (trisomy 21) where PAH can be caused by the high incidence of left-to-right shunt, upper airway obstructions and sleep apnoea. Other disorders where PAH has been described are congenital heart disease (CHD), 22q11 deletion syndrome (DiGeorge), VACTERL syndrome, CHARGE syndrome, Scimitar syndrome, Noonan syndrome, chromosomal anomalies associated with congenital diaphragmatic hernia, Cantu syndrome, autoimmune polyendocrine syndrome, mitochondrial disorders including mitochondrial encephalopathy lactic acidosis and stroke-like episodes, Gaucher disease, and glycogen storage diseases (GSDI and GSDIII). Establishing the underlying molecular genetic defect in the majority of these syndromes can be beneficial in determining the exact diagnosis and estimating prognosis, clinical follow-up as well as treatment strategies. This recommendation does not focus on the molecular genetics of these syndromes nor does it recommend testing of PAH-associated genes in these patients.

\section{Genetic testing in PAH}

Considering the possible hereditary nature of $\mathrm{PAH}$, identification of a child with idiopathic PAH will initiate concerns of disease susceptibility in other family members, including the parents. Clinical evaluation of patient and family members will not necessarily establish the aetiology of IPAH and cannot define their risk to develop PAH later in life. The latter, like in other hereditary diseases, causes significant distress for the family. Identification of the genetic background enables effective family member testing and risk stratification among relatives. Considering the relatively high penetrance, especially among women, identification of the causative mutation allows effective clinical monitoring of those individuals at increased risk for the disease. Similarly, negative testing in family members following the molecular diagnosis for an index patient reduces stress burden in family members and allows discontinuation of future clinical monitoring in these individuals. This approach could therefore enable the establishment of cost-effective monitoring and follow-up programmes. ${ }^{2}$ Follow-up strategies for mutation carrier relatives have to be evaluated individually based on the age and sex of the family member, and existing family history and disease characteristics.

Genetic counselling is recommended for families with children suffering from a genetic disorder. Genetic counselling has to elucidate to the patient and family the rationale for genetic testing and describe the expected yield of genetic testing. Counselling also includes addressing the complex issues of incomplete penetrance and the possibility of finding variants that currently are classified as variants of unknown significance. All variant classification scenarios in genetic testing have to be clear for the referring physician. ${ }^{17}$ Different end points may affect the future surveillance of family members. It is also recommended to discuss concerns about genetic discrimination, and psychosocial issues of guilt and blame that accompany genetically based diseases. Although there have been concerns that predictive genetic testing in clinically unaffected children and adults could provoke anxiety and distress, current studies suggest instead that it can provide reassurance, lessen uncertainty and promote a sense of control. ${ }^{18}$ Genetic counselling should be provided by a healthcare professional with experience in genetics and PAH. Determining the best age for testing the asymptomatic children has to be evaluated separately in every family based on the family history and family's requests after counselling. An algorithm for genetic testing of a child with IPAH or HPAH has been described in figure 1 .

An important part of the counselling process is to establish an inclusive family history and determine the clinical features of the index patient. This allows comprehensive description of the disease phenotype and will facilitate interpretation of genetic test results. Any family history of HHT-like symptoms could point towards a mutation in the ACVRL1 or ENG gene, whereas at the same time a negative family history of HHT, does not rule out the possibility of disease-causing mutation in the ACVRL1 gene. On the contrary, suspicion of PVOD, especially if recessive inheritance is possible, suggests mutations in the EIF2AK4 gene. Although BMPR2 and ACVRL1 are currently the major diseasecausing genes in PAH, any patient with IPAH or HPAH could harbour a mutation in any of the other known PAH genes. As the number of disease-causing genes is expanding there is increasing rationale to select more effective high-throughput next generation sequencing (NGS) strategies instead of gene-specific direct sequencing (Sanger sequencing). NGS-based applications are already enabling more cost-effective, comprehensive and faster analysis of causative genes in diagnostic setting. As the field and 
Figure 1 Algorithm for applying genetic testing for a child with IPAH or HPAH, and family members. (-) negative test result and $(+)$ positive test result with identification of pathogenic or likely pathogenic mutation. If NGS-based comprehensive consider gene-specific testing for $B M P R 2$ and $A C V R L 1$ genes as first-line diagnostics. *If genetic test reveals a mutation classified as likely pathogenic consider new genetic counselling in 5 years to confirm classification and validity of applied follow-up strategy. \# Follow-up strategies for mutation carrier relatives have to be evaluated individually based on the age and sex of the family member, and existing family history and disease characteristics. HPAH, hereditary pulmonary arterial hypertension; IPAH, idiopathic pulmonary arterial hypertension; NGS, next generation sequencing; $\mathrm{PAH}$, pulmonary arterial hypertension. analysis of PAH genes is not available

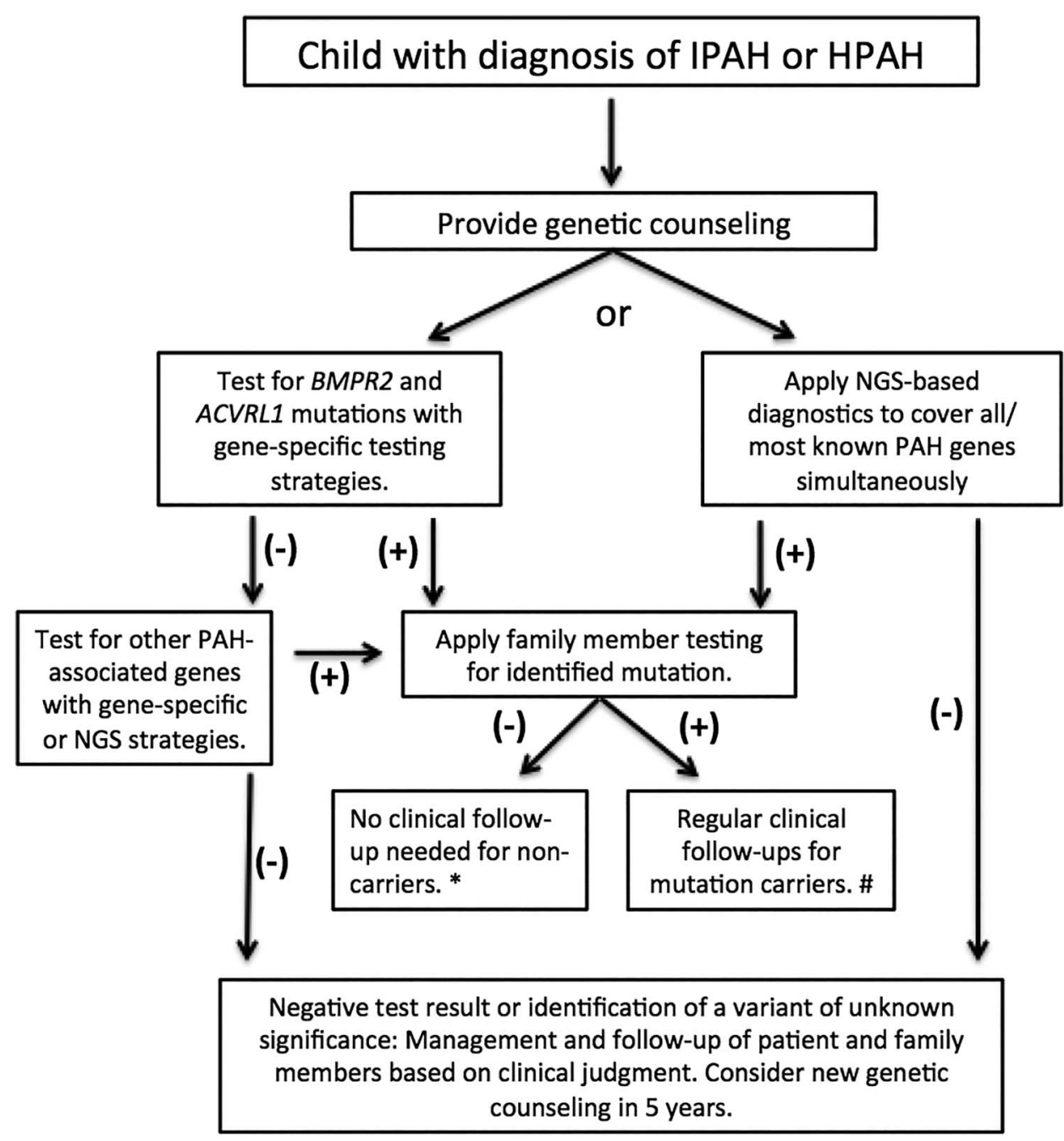

technologies are evolving rapidly there is increasing pressure for the referring physician to select optimal diagnostic tools to ensure high-end test quality and interpretation of test results. NGS strategies are already enabling the analysis of all nine genes mentioned in this statement with the same costs as single gene analysis using conventional technologies. Multigene strategies will provide simultaneous full analysis of BMPR2 and ACVRL1 and can include the analysis of other genes causative for PAH as well as potential new candidates. Adapting this strategy will allow for increasing diagnostic power but may also accumulate variant data impacting future diagnostics. Today's genetic diagnostics is increasingly based on public databases, which accumulate variant information from diagnostics and research. It is becoming a standard procedure in modern genetic diagnostics to publish variant data and classifications in public databases such as ClinVar (http://www.ncbi.nlm.nih.gov/clinvar/).

The use of biomarkers in paediatric PAH

Existing and potential biomarkers in PAH relate to heart failure, inflammation, haemostasis, vascular remodeling and endothelial cell-smooth muscle cell dysfunctions. In current PAH guidelines, brain natriuretic peptide (BNP) and N-terminal fragment of pro-BNP (NT-proBNP) have been recommended. ${ }^{19}$ Other prognostic markers such as growth differentiation factor-15, osteopontin and red cell distribution width, are considered emerging biomarkers of PAH. Anaemia, hypocapnia, elevated uric acid and $\mathrm{C}$ reactive protein levels are unspecific markers of disease severity. Certain chemokines, matrix metalloproteases and apelin have been linked to the vascular pathology in $\mathrm{PAH}$ and are considered as potential biomarkers. Circulating endothelial cells (CECs) and endothelial progenitor cells (EPCs) have received much attention as markers of disease activity but with controversial findings. Circulating microparticles and microRNAs have also been suggested. The majority of the studies in this field have been done with adults and the role of most candidate biomarkers in paediatric PAH is unknown. As in adults, the strongest evidence is on the use of BNP and NT-proBNP in monitoring disease severity in paediatric PAH. Biomarkers in children with PAH are likely to be impacted by additional factors, more so than in adult patients with PAH. These factors include degree of physical activity, age and/or developmental stage, gender differences, puberty and nutritional status. Although several promising markers have been identified over the past few years, the development of more specific markers, standardisation and prospective validation are warranted, also in paediatric PAH.

Natriuretic peptides and other plasma protein biomarkers $\mathrm{PAH}$ that natriuretic peptides have prognostic value. ${ }^{20-22}$ In these studies, elevated natriuretic peptides correlated with poor haemodynamics and increased mortality. The natriuretic peptides atrial natriuretic peptide (ANP) and BNP are synthesised in the atrial and the ventricular myocardium. These peptides There is solid evidence in adult and paediatric patients with 
regulate natriuresis, diuresis and vasomotor tone. Atrial natriuretic peptide and BNP are elevated in association with pressure overload in order to reduce the workload of the heart and therefore have been found to be elevated in PAH. BNP is produced as a 108 amino acid prohormone, which is cleaved and secreted as a biological active sequence (BNP-32) and an inactive NT-proBNP peptide. BNP-32 has a short half-life of around $20 \mathrm{~min}$ and NT-proBNP a longer half-life of several hours, but both fragments are cleared by the kidney and influenced by renal function. A recent study elucidates that NT-proBNP levels correlated with haemodynamic parameters and outcome in PAH regardless of renal function. ${ }^{23}$ Large variability of normal NT-proBNP levels at different ages during childhood has to be acknowledged when applied in paediatric PAH. ${ }^{24}$

Cardiac troponin $\mathrm{T}$ has also been shown as a significant biomarker in PAH correlating independently with mortality. ${ }^{2}$ Evidence in paediatric population is lacking. Another study on children with CHD and PAH revealed elevated serum levels of soluble intracellular adhesion molecule $1 .^{3}$ In addition growth differentiation factor-15, galectin-3, endothelins and $\mathrm{C}$ reactive protein are promising as biomarkers in adult $\mathrm{PAH}$, at least with respect to inflammation. A large number of other inflammatory proteins have also been suggested as candidates for biomarkers in PAH. As in genetics, the need for a comprehensive approach targeting large number of PAH-specific analytes is useful to optimise use of protein biomarkers in everyday clinical practice.

\section{Uric acid}

Uric acid is the final degradation product of purines and an endogenous free radical scavenger that is elevated following tissue ischaemia and hypoxia. Raised levels have been described in chronic obstructive pulmonary disease, ${ }^{8}$ chronic heart failure ${ }^{9}$ and cyanotic CHD. ${ }^{10}$ This was underlined by reductions of uric acid levels in response to chronic vasodilator therapy. Potential confounding factors, however, include renal dysfunction and diuretic use and hence uric acid levels must be interpreted in the context of these factors. The role of uric acid in monitoring PAH severity and treatment responses may be useful although further studies are needed. ${ }^{11} 25$

\section{Circulating endothelial cells}

Circulating endothelial cells (CECs) have been established as noninvasive markers for vascular damage, remodeling and dysfunction. CECs have been measured in children with IPAH and PAH associated with CHD, before and after treatment. ${ }^{26}$ Rising CEC counts predicted clinical deterioration, suggesting that CECs may be an important tool to evaluate prognosis. ${ }^{26,}{ }^{27}$ Another study reported elevated levels of CECs associated with reversible APAH-CHD in children. ${ }^{27}$ These studies $^{26,27}$ involved analysis of soluble biomarkers, and determined that markers of angiogenic cytokines, inflammation or endothelial microparticles were not able to predict PAH reversibility. These studies suggest that circulating CECs could be a potential biomarker in the assessment of paediatric PAH. On the other hand, some studies have failed to establish any correlation between CECs and PAH. ${ }^{6}{ }^{28}$ The lack of standards for cell isolation and characterisation methods as well as differences in the pathological mechanisms of the investigated patients may contribute to the existing discrepancies.

\section{Conclusions}

Emerging evidence is accumulating supporting the use of genetic testing and blood biomarkers in paediatric $\mathrm{PAH}$ to individualise diagnostics, ${ }^{32}$ prognostics and therapy. ${ }^{33}$ In addition, genetic testing supports efficient risk stratification in family members. As the number of genes and biomarkers associated with PAH is accumulating, we need more comprehensive diagnostic approaches in both fields. In addition to new diagnostic technologies, advanced experimental approaches and patient registries including multicentre approaches are needed, to strengthen current evidence and develop future recommendations.

\section{Author affiliations}

${ }^{1}$ Department of Paediatric Cardiology, University of Heidelberg, Heidelberg, Germany ${ }^{2}$ Perinatal Center Grosshadern, Dr. von Haunersches Children's Hospital, LudwigMaximilians-University, Munich, Germany

${ }^{3}$ Department of Clinical Physiolology, University Hospital Helsinki, University of Helsinki, Helsinki, Finland

${ }^{4}$ Blueprint Genetics, Biomedicum Helsinki, Helsinki, Finland

${ }^{5}$ Paediatric Heart Centre, University Hopsital of Giessen and Marburg, Giessen, Germany

${ }^{6}$ Department of Paediatric Cardiology and Critical Care, Hannover Medical School, Hannover, Germany

${ }^{7}$ Department of Paediatric Cardiology, Childrens Hospital Helsinki, University of Helsinki, Helsinki, Finland

Funding CA currently receives grant funding from Stiftung Kinder Herz (2511-1013-001) and Behring Röntgen Stiftung (59-0018). GH currently receives grant support from the German Research Foundation (DFG; HA4348/2-1),

Fördergemeinschaft deutsche Kinderherzzentren (W-H-001-2014), and Stiftung KinderHerz (2511-6-13-011).

This Heart supplement was produced with support from an unrestricted educational grant from Actelion Pharmaceuticals Germany GmbH, Bayer Pharma AG and Pfizer Inc. None of these organisations had any influence on the composition of the writing group or the content of the articles published in this supplement. Open Access publication of this article was sponsored by Actelion Pharmaceuticals Germany GmbH.

Competing interests JP, MG, $\mathrm{AH}, \mathrm{CA}$ and $\mathrm{GH}$ indicate no conflicts of interests related to the content of this article. TPA and JWK are co-founders and Medical Directors of Blueprint Genetics, which develops technologies for genetic diagnostics and interpretation of genetic information.

Relationships with industry (RWI) JP, MG, AH, CA, and GH indicate no relevant relationship with industry. TPA and JWK hold stock and have received partial salary from Blueprint Genetics.

Provenance and peer review Commissioned; externally peer reviewed.

Open Access This is an Open Access article distributed in accordance with the Creative Commons Attribution Non Commercial (CC BY-NC 4.0) license, which permits others to distribute, remix, adapt, build upon this work non-commercially, and license their derivative works on different terms, provided the original work is properly cited and the use is non-commercial. See: http://creativecommons.org/ licenses/by-nc/4.0/

\section{REFERENCES}

1 Ackerman MJ, Priori SG, Willems S, et al. HRS/EHRA expert consensus statement on the state of genetic testing for the channelopathies and cardiomyopathies: this document was developed as a partnership between the Heart Rhythm Society (HRS) and the European Heart Rhythm Association (EHRA). Europace 2011;13:1077-109.

2 Ingles J, McGaughran J, Scuffham PA, et al. A cost-effectiveness model of genetic testing for the evaluation of families with hypertrophic cardiomyopathy. Heart 2012;98:625-30.

3 Soubrier F, Chung WK, Machado R, et al. Genetics and genomics of pulmonary arterial hypertension. J Am Coll Cardiol 2013;62:D13-21.

4 Larkin EK, Newman JH, Austin ED, et al. Longitudinal analysis casts doubt on the presence of genetic anticipation in heritable pulmonary arterial hypertension. Am J Respir Crit Care Med 2012;186:892-6.

5 Harrison RE, Berger R, Haworth SG, et al. Transforming growth factor-beta receptor mutations and pulmonary arterial hypertension in childhood. Circulation 2005; 111:435-41.

6 Chida A, Shintani M, Yagi H, et al. Outcomes of childhood pulmonary arterial hypertension in BMPR2 and ALK1 mutation carriers. Am I Cardiol 2012;110:586-93.

7 Harrison RE, Flanagan JA, Sankelo $M$, et al. Molecular and functional analysis identifies ALK-1 as the predominant cause of pulmonary hypertension related to hereditary haemorrhagic telangiectasia. J Med Genet 2003;40:865-71.

8 Drake KM, Zygmunt D, Mavrakis L, et al. Altered MicroRNA processing in heritable pulmonary arterial hypertension: an important role for Smad-8. Am J Respir Crit Care Med 2011;184:1400-8.

9 Chida A, Shintani M, Nakayama T, et al. Missense mutations of the BMPR1B (ALK6) gene in childhood idiopathic pulmonary arterial hypertension. Circ J 2012;76:1501-8. 
10 Ma L, Roman-Campos D, Austin ED, et al. A novel channelopathy in pulmonary arterial hypertension. N Engl J Med 2013;369:351-61.

11 Chida A, Shintani M, Matsushita Y, et al. Mutations of NOTCH3 in childhood pulmonary arterial hypertension. Mol Genet Genomic Med 2014;2:229-39.

12 Eyries M, Montani D, Girerd B, et al. EIF2AK4 mutations cause pulmonary veno-occlusive disease, a recessive form of pulmonary hypertension. Nat Genet 2014:46:65-9.

13 Koehler R, Grünig E, Pauciulo MW, et al. Low frequency of BMPR2 mutations in a German cohort of patients with sporadic idiopathic pulmonary arterial hypertension. J Med Genet 2004;41:e127.

14 Austin ED, Cogan JD, West JD, et al. Alterations in oestrogen metabolism: implications for higher penetrance of familial pulmonary arterial hypertension in females. Eur Respir J 2009;34:1093-9.

15 Sztrymf B, Coulet F, Girerd B, et al. Clinical outcomes of pulmonary arterial hypertension in carriers of BMPR2 mutation. Am J Respir Crit Care Med 2008;177:1377-83.

16 Girerd B, Montani D, Coulet F, et al. Clinical outcomes of pulmonary arterial hypertension in patients carrying an ACVRL1 (ALK1) mutation. Am J Respir Crit Care Med 2010;181:851-61.

17 Richards CS, Aziz N, Bale S, et al. Standards and guidelines for the interpretation of sequence variants: a joint consensus recommendation for the American College of Medical Genetics and Genomics and the Association for Molecular Pathology. Genet Med 2015;17:405-23.

18 Aatre RD, Day SM. Psychological issues in genetic testing for inherited cardiovascular diseases. Circ Cardiovasc Genet 2011;4:81-90.

19 Vattulainen S, Aho J, Salmenpera P, et al. Accurate genetic diagnosis of Finnish pulmonary arterial hypertension patients using oligonucleotide-selective sequencing Mol Genet Genomic Med 2015;3:354-62.

20 Task Force for Diagnosis and Treatment of Pulmonary Hypertension of European Society of Cardiology (ESC), European Respiratory Society (ERS), International Society of Heart and Lung Transplantation (ISHLT), et al. Guidelines for the diagnosis and treatment of pulmonary hypertension. Eur Respir J 2009:34:1219-63.

21 Van Albada ME, Loot FG, Fokkema R, et al. Biological serum markers in the management of paediatric pulmonary arterial hypertension. Pediatr Res 2008;63:321-7.

22 Bernus A, Wagner BD, Accurso F, et al. Brain natriuretic peptide levels in managing paediatric patients with pulmonary arterial hypertension. Chest 2009;135:745-51.
23 Lammers AE, Hislop AA, Haworth SG. Prognostic value of B-type natriuretic peptide in children with pulmonary hypertension. Int J Cardiol 2009;135:21-6.

24 Harbaum L, Hennigs JK, Baumann HJ, et al. N-terminal pro-brain natriuretic peptide is a useful prognostic marker in patients with pre-capillary pulmonary hypertension and renal insufficiency. PLOS ONE 2014;9:e94263.

25 Dhaun N, Vachiery J-L, Benza RL, et al. Endothelin antagonism and uric acid levels in pulmonary arterial hypertension: clinical associations. J Heart Lung Transplant 2014;33:521-7.

26 Levy M, Bonnet D, Mauge L, et al. Circulating endothelial cells in refractory pulmonary hypertension in children: markers of treatment efficacy and clinical worsening. PLOS ONE 2013;8:e65114.

27 Smadja DM, Gaussem P, Mauge L, et al. Comparison of endothelial biomarkers according to reversibility of pulmonary hypertension secondary to congenital heart disease. Pediatr Cardiol 2010;31:657-62.

28 Schiavon M, Fadini GP, Lunardi F, et al. Increased tissue endothelial progenitor cells in end-stage lung diseases with pulmonary hypertension. J Heart Lung Transplant 2012;31:1025-30.

29 Badesch DB, Abman SH, Simonneau G, et al. Medical theraphy for pulmonary arterial hypertension: updated ACCP evidence-based clinical practice guidelines. Chest 2007;131:1917-28.

30 McLaughlin VV, Archer SL, Badesch DB, et al. ACCF/AHA 2009 expert consensus document on pulmonary hypertension: a report of the American College of Cardiology Foundation Task Force on Expert Consensus Documents and the American Heart Association: developed in collaboration with the American College of Chest Physicians, American Thoracic Society, Inc., and the Pulmonary Hypertension Association. Circulation 2009;119:2250-94.

31 Hansmann G, Apitz C, Abdul-Khaliq H, et al. Executive summary. Expert consensus statement on the diagnosis and treatment of paediatric pulmonary hypertension. The European paediatric pulmonary vascular disease network, endorsed by ISHLT and DGPK. Heart 2016;102:ii86-100.

32 Lammers A, Apitz C, Zartner $\mathrm{P}$, et al. Diagnostics, monitoring and outpatient care in children with suspected pulmonary hypertension/paediatric pulmonary hypertensive vascular disease. Expert consensus statement on the diagnosis and treatment of paediatric pulmonary hypertension. The European paediatric pulmonary vascular disease network, endorsed by ISHLT and DGPK. Heart 2016;102:ii1-13.

33 Hansmann G, Apitz C. Treatment of children with pulmonary hypertension and cardiac dysfunction. Expert consensus statement on the diagnosis and treatment of paediatric pulmonary hypertension. The European paediatric pulmonary vascular disease network, endorsed by ISHLT and DGPK. Heart 2016;102:ii67-85. 